\title{
Profil de l'ionogramme sanguin chez les enfants brûlés du Centre Hospitalier Universitaire Pédiatrique Charles de Gaulle de Ouagadougou (Burkina Faso)
}

\author{
Fabienne Marie SOUDRE ${ }^{1,2 *}$, Alice KIBA ${ }^{1,3}$, Arnaud KOURAOGO ${ }^{1,4}$, Raoul KARFO ${ }^{1,4}$, \\ Salam TIENDREBEOGO ${ }^{1,2}$, Augustin KYETEGA ${ }^{2}$, Dieudonné SIRE ${ }^{2}$, Elie KABRE ${ }^{1,5}$ \\ et Jean SAKANDE ${ }^{1,4}$ \\ ${ }^{1}$ Unité de Formation et de Recherche en Sciences de la Santé, Université Joseph Ki-Zerbo, 03 BP 7021, \\ Ouagadougou, Burkina Faso. \\ ${ }^{2}$ Centre Hospitalier Universitaire Pédiatrique Charles de Gaulle, 01 BP 1198, Ouagadougou, Burkina Faso. \\ ${ }^{3}$ Centre Hospitalier Universitaire de Tengandogo, 11 BP 104, Ouagadougou, Burkina Faso. \\ ${ }^{4}$ Centre Hospitalier Universitaire Yalgado Ouédraogo, 01 BP 5234, Ouagadougou, Burkina Faso. \\ ${ }^{5}$ Laboratoire National de Santé Publique, 09 BP 24, Ouagadougou, Burkina Faso. \\ *Auteur correspondant; E-mail : fabysoudre@gmail.com; Tél. : +22670455106
}

Received: 04-02-2021

Accepted: $23-04-2021$

Published: 30-04-2021

\section{RESUME}

Les brûlures de l'enfant peuvent être à l'origine de troubles ioniques graves, entrainant une morbimortalité importante. Ce travail avait pour objectif de décrire les perturbations de l'ionogramme sanguin chez les enfants brûlés, afin de contribuer à l'amélioration des soins. Il s'est agi d'une étude transversale rétrospective allant du 01 janvier 2017 au 30 juin 2019. Ont été inclus les enfants hospitalisés dans le service de chirurgie du CHU pédiatrique Charles de Gaulle pour brûlure et chez lesquels un ionogramme sanguin a été réalisé. L'étude a porté sur 212 enfants, avec un âge moyen de 38,52 mois et un sex ratio de 1,12. La surface corporelle brûlée moyenne était de $26,60 \%$ et le délai moyen d'admission de 5,71 heures. La mortalité était de $16,98 \%$. L'ionogramme sanguin à l'entrée a retrouvé une hyponatrémie (27,88\%), une hypobicarbonatémie $(53,95 \%)$, une hypoprotidémie $(26,67 \%)$ et une hyperchlorémie $(53,59 \%)$. Au contrôle, ces troubles ioniques étaient toujours présents. S'y sont associées une hypocalcémie (30,43\%), une hypomagnésémie (27,27\%) et une hyperphosphatémie (28,79\%). La natrémie, la bicarbonatémie et la protidémie à l'entrée étaient significativement plus basses chez les patients décédés. Les brûlures de l'enfant sont fréquentes. Leur évolution est marquée par des perturbations de l'ionogramme sanguin et une mortalité élevée.

(C) 2021 International Formulae Group. All rights reserved.

Mots clés : Brûlure, Ionogramme sanguin, Enfants, Burkina Faso.

\section{Blood ionogram profile in burned children at the Charles de Gaulle University Pediatric Hospital of Ouagadougou (Burkina Faso)}

\begin{abstract}
Burns in children can be the cause of serious ionic disorders, leading to significant morbidity and mortality. The aim of this study was to describe the disturbances in the blood ionogram of burnt children, in order to contribute to the improvement of care. This was a retrospective cross-sectional study from 01 January 2017 to
\end{abstract}


30 June 2019. Children hospitalised in the surgery department of the Charles de Gaulle Pediatric University Hospital for burns and in whom a blood ionogram was carried out were included. Our study involved 212 children, with an average age of 38.52 months and a sex ratio of 1.12. The average body surface area burned was $26.60 \%$ and the average admission time was 5.71 hours. Mortality was $16.98 \%$. The blood ionogram at the entrance showed hyponatremia (27.88\%), hypobicarbonatremia (53.95\%), hypoprotidemia $(26.67 \%)$ and hyperchloremia (53.59\%). At the control, these ionic disorders were still present. They were associated with hypocalcaemia (30.43\%), hypomagnesemia (27.27\%) and hyperphosphatemia (28.79\%). Natremia, bicarbonateemia and protidemia were significantly lower on admission in patients who died in the course of the evolution. Childhood burns are frequent at the CHUP-CDG; their evolution is marked by disturbances of the blood ionogram and high mortality.

(C) 2021 International Formulae Group. All rights reserved.

Keywords: Burns, Blood Ionogram, Children, Burkina Faso.

\section{INTRODUCTION}

Les brûlures constituent un problème mondial de santé publique, occasionnant, selon les estimations de l'Organisation mondiale de la santé (OMS), 180000 décès par an (OMS, 2020). Elles se définissent comme une destruction partielle ou totale du revêtement cutané ou des tissus sous-jacents par un agent thermique, électrique, chimique ou par des radiations ionisantes. Il s'agit d'un phénomène local à retentissement général pouvant mettre en jeu le pronostic vital. Chez l'enfant, les liquides chauds en sont la cause dans près de 75\% des cas (Peck, 2011; Gülhan et al., 2019; Santiso et al., 2020). La gravité d'une brûlure est définie selon la surface corporelle brûlée (SCB), la profondeur, la localisation, le terrain (âge et antécédents) et l'existence de lésions associées (lésions pulmonaires par inhalation ou autres traumatismes) (Wassermann, 2002; Ryder et al., 2020; Zhang et al., 2020).

Avec les progrès de la réanimation et la création de Centres de traitement de brûlés, la mortalité est en baisse constante dans les pays à revenus élevés. Ainsi, en France métropolitaine, le rapport annuel de la Société française d'étude et de traitement des brûlures (SFETB) indiquait une mortalité globale de 2,1\% (SFETB, 2008). Cependant la mortalité reste élevée dans les pays à faibles revenus (dont la plupart des Etats africains) où survient la majorité des décès annuels dus aux brûlures. Ainsi au Mali en 2007 Bagayoko (2007) notait une mortalité de 17,1\%, Fadeyibi (Fadeyibi et al., 2011) au Nigeria 29,9\% et Beyiha (Beyiha et al., 2000) au Cameroun 17,8\%. Au Burkina
Faso (BF), le centre hospitalier universitaire Sourô Sanou (CHU-SS) de Bobo-Dioulasso a noté de 2009 à 2013 une fréquence en hospitalisation de 5,1\% avec une mortalité de $71 \%$ (Guibla, 2015). Le centre hospitalier universitaire pédiatrique Charles de Gaulle (CHUP-CDG) de Ouagadougou a noté de 2005 à 2010 une fréquence en hospitalisation de $4,78 \%$ avec une mortalité globale de 20,2\% (Konvolbo, 2011) et de 2011 à 2017 une fréquence en hospitalisation de $6,89 \%$ avec une mortalité de 20,76\% (Guedehounsou, 2015).

La physiopathologie de la brûlure fait état d'une phase initiale dominée par les troubles hydroélectrolytiques, dont les plus courants sont l'hyponatrémie, l'hyperkaliémie, l'hypocalcémie, l'hypophosphorémie et l'hypoprotidémie (Ramos, 2000; HauhouotAttoungbre et al., 2005). Ces troubles sont présents et majorés avec l'étendue de la surface corporelle brûlée (SCB); or chez l'enfant, le pronostic vital est en jeu dès que la SCB dépasse 10\% (Wassermann, 2002; Minkandé et al., 2004; Bargues et al., 2009). C'est ainsi que le CHUP-CDG a noté de 2011 à 2017 une mortalité de 69,38\% chez les enfants ayant une SCB supérieure à 30\% (Guedehounsou, 2015).

En outre, sur le plan national, aucune étude n'a été consacrée aux perturbations des paramètres hydroélectrolytiques au cours de la prise en charge des brûlures de l'enfant. Ce travail a donc été mené afin de mettre en exergue le profil des électrolytes sanguins dans les brûlures de l'enfant au CHUP-CDG, dans l'optique de contribuer à l'amélioration de la prise en charge. 


\section{MATERIEL ET METHODES}

\section{Cadre, type et période d'étude}

Il s'est agi d'une étude transversale à visée descriptive et analytique, allant du 1er janvier 2017 au 30 juin 2019. Les données de 2017 et 2018 ont été collectées rétrospectivement; tandis que celles de 2019 l'ont été de manière prospective. L'étude a été menée au CHUP-CDG de Ouagadougou au Burkina Faso, plus particulièrement dans le service de chirurgie et l'unité de biochimie du laboratoire d'analyses biomédicales.

\section{Patients}

Ont été inclus dans notre étude, tous les enfants hospitalisés dans le service de chirurgie du CHUP-CDG durant la période du $1^{\mathrm{er}}$ janvier 2017 au 30 juin 2019 et répondant aux critères suivants : brûlure du deuxième ou troisième degré, délai entre la brûlure et l'admission à l'hôpital inférieur ou égal à 24 heures et dossier clinique exploitable. Cependant, les sujets souffrant de maladies ou prenant des traitements susceptibles de perturber l'ionogramme sanguin n'ont pas été retenus.

\section{Méthodes}

Les données socio-démographiques et cliniques des patients ont été obtenues à partir des registres d'hospitalisation et des dossiers médicaux disponibles au service de chirurgie.
Quant aux données biochimiques, nous avons consulté le registre des résultats de l'unité de biochimie. Ces résultats ont été obtenus par dosage des différents paramètres de l'ionogramme sanguin sur un prélèvement de sang veineux sur tube sec, centrifugé à 3500 tours/minute pendant cinq minutes et duquel a été prélevé le sérum. Les méthodes de dosage, ainsi que les valeurs normales de ces paramètres sont présentées dans le Tableau 1. Chez chaque patient, au moins deux prélèvements ont été réalisés: le premier à l'admission ; puis des prélèvements de contrôle ont été effectués (dont le premier dans un délai moyen de sept jours). Pour notre étude, nous avons retenu les résultats du prélèvement d'entrée et ceux du premier contrôle.

Toutes les données ont été recueillies sur une fiche de collecte établie à cet effet et traitées dans le strict respect de la confidentialité.

\section{Traitement et analyse des données}

Les fiches remplies ont été saisies sur ordinateur et les données analysées à l'aide du logiciel Stata version 13.0. Les graphiques et tableaux ont été obtenus grâce au logiciel Excel 2016. Les comparaisons de moyennes ont été effectuées en utilisant le test $\mathrm{t}$ de Student. Le seuil de signification statistique a été fixée à $\mathrm{p}<0,05$.

Tableau 1 : Méthodes de dosage et valeurs normales des paramètres de l'ionogramme sanguin au Centre Hospitalier Universitaire Pédiatrique Charles de Gaulle.

\begin{tabular}{llrrr}
\hline $\begin{array}{l}\text { Paramètres } \\
\text { de l'ionogramme } \\
\text { sanguin }\end{array}$ & Méthode de dosage & \multicolumn{3}{c}{ Valeurs normales } \\
\cline { 3 - 5 } & & $\begin{array}{r}\text { Nouveau-né } \\
\mathbf{( 0 - 2 8 j o u r s )}\end{array}$ & $\begin{array}{r}\text { Nourrisson } \\
(\mathbf{2 9} \text { jours-30 mois) }\end{array}$ & $\begin{array}{r}\text { Enfant } \\
\mathbf{( 3 0} \text { mois-15ans) }\end{array}$ \\
\hline Sodium $(\mathrm{mmol} / \mathrm{L})$ & Potentiométrie & $130-145$ & $130-145$ & $135-145$ \\
Potassium (mmol/L) & Potentiométrie & $3,5-7$ & $3,5-7$ & $3,5-5,5$ \\
Chlore $(\mathrm{mmol} / \mathrm{L})$ & Potentiométrie & $95-110$ & $95-110$ & $98-105$ \\
Calcium (mmol/L) & Arsénazo III & $2,0-3,0$ & $2,0-3,0$ & $2,2-2,5$ \\
Magnésium (mmol/L) & Bleu de xylidyle & $0,6-0,8$ & $0,6-0,8$ & $0,6-1$ \\
Phosphore (mmol/L) & Molybdate & $1,30-2,25$ & $1,30-2,25$ & $1,3-1,85$ \\
Bicarbonates (mmol/L) & Enzymatique & $14-27$ & $14-27$ & $20-28$ \\
Protéines totales (g/L) & Biuret & $45-70$ & $45-70$ & $60-80$ \\
\hline
\end{tabular}




\section{RESULTATS}

Du 1er janvier 2017 au 30 juin 2019, 7908 patients ont été admis dans le service de chirurgie du CHUP-CDG parmi lesquels il y avait 384 brûlés, soit $4,85 \%$ des hospitalisations. Pour notre étude, 212 enfants brûlés remplissaient les critères et ont été inclus. La répartition des cas de brûlures en fonction du mois et de l'année est présentée dans la Figure 1, avec des pics constatés aux mois de janvier et février.

\section{Caractéristiques socio-démographiques et cliniques des patients}

Les caractéristiques sociodémographiques et cliniques des patients sont présentées dans le Tableau 2. L'âge moyen était de 38,52 mois (avec des extrêmes allant de 2 mois à 15 ans) et le sex-ratio $\mathrm{H} / \mathrm{F}$ de 1,12. Tous les enfants avaient été victimes de brûlure thermique, avec comme principal agent causal l'eau chaude $(69,08 \%)$. Les autres agents étaient représentés par les flammes $(15,94 \%)$, la sauce $(6,28 \%)$, l'huile $(5,32 \%)$ et la bouillie $(3,38 \%)$; soit un total de $30,92 \%$. Le délai moyen d'admission était de 5,71 heures, avec une surface corporelle brûlée moyenne de $26,60 \%$ (allant de $4 \%$ et $95 \%$ ) et une mortalité de $16,98 \%$.

\section{Profil des perturbations de l'ionogramme sanguin}

Les fréquences des perturbations de l'ionogramme sanguin retrouvées à l'admission du patient, puis lors du premier contrôle, sont présentées dans le Tableau 3. A l'entrée, les principales perturbations étaient représentées par l'hyponatrémie $(27,88 \%)$, l'hypobicarbonatémie

l'hypoprotidémie $\quad(26,67 \%) \quad$ et l'hyperchlorémie $\quad(53,59 \%)$. Au contrôle, l'hyponatrémie $\quad(24,64 \%)$, l'hypobicarbonatémie $\quad(32,00 \%)$, l'hypoprotidémie $\quad(62,32 \%) \quad$ et l'hyperchlorémie $(39,71 \%)$ étaient toujours présentes. S'y sont associées, une hypocalcémie $(30,43 \%)$, une hypomagnésémie $(27,27 \%)$ et une hyperphosphatémie $(28,79 \%)$.

Profils des paramètres d'entrée de l'ionogramme sanguin en fonction des caractéristiques cliniques des patients

Les Tableaux 4 et 5 présentent les profils des paramètres de l'ionogramme sanguin à l'entrée des patients, en fonction de leurs caractéristiques cliniques: d'abord en fonction du délai d'admission et de l'évolution clinique des patients; puis de la surface corporelle brûlée et de la profondeur de la brûlure.

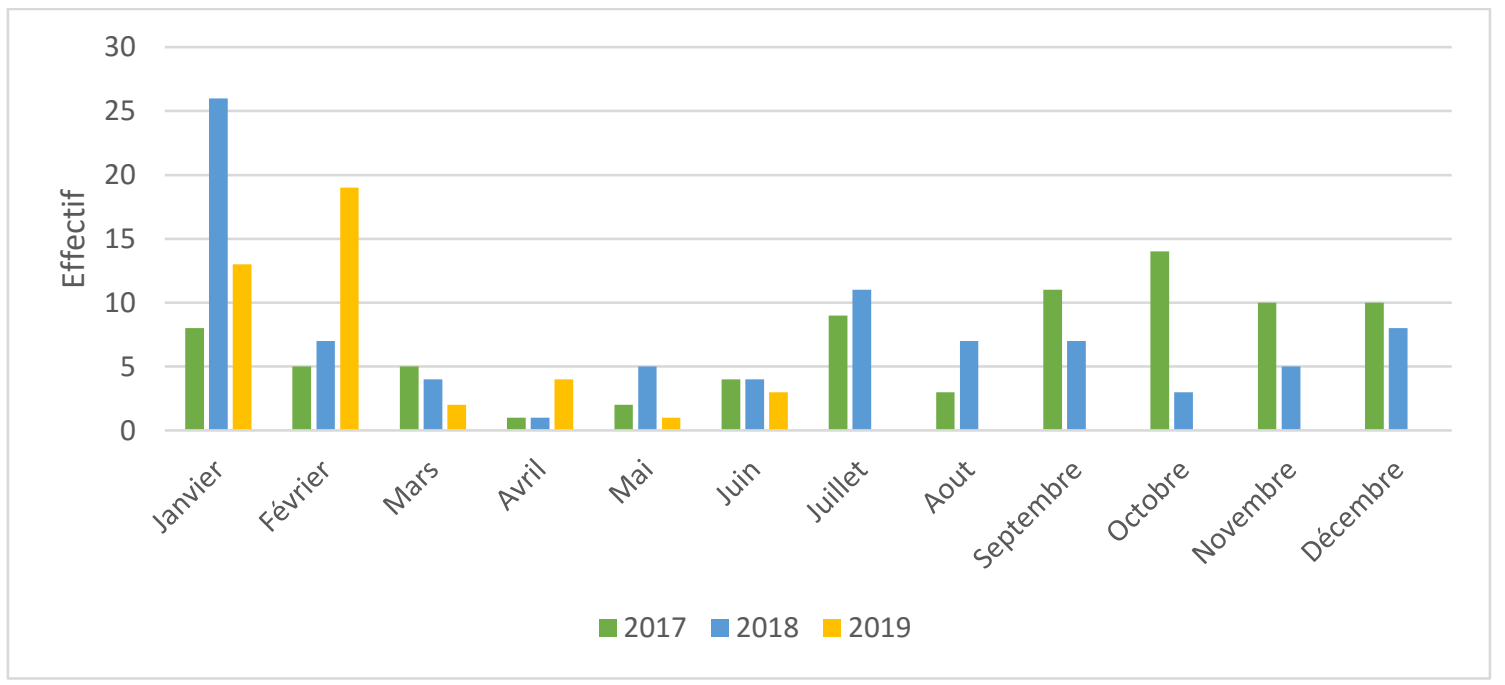

Figure 1 : Répartition des cas de brûlures en fonction du mois et de l'année. 
Tableau 2 : Répartition des patients en fonction des caractéristiques socio-démographiques et cliniques.

\begin{tabular}{|c|c|c|c|}
\hline Caractéristiques & & Effectif & Pourcentage (\%) \\
\hline \multicolumn{4}{|c|}{ Caractéristiques socio-démographiques } \\
\hline \multirow{2}{*}{$\begin{array}{l}\text { Age (mois) } \\
(n=212)\end{array}$} & [0 à 30] & 119 & 56,13 \\
\hline & ] 30 à 180] & 93 & 43,87 \\
\hline \multirow{2}{*}{$\begin{array}{l}\text { Sexe } \\
(n=212)\end{array}$} & Masculin & 112 & 52,83 \\
\hline & Féminin & 100 & 47,17 \\
\hline \multirow{2}{*}{$\begin{array}{l}\text { Agent causal } \\
(n=207)\end{array}$} & Eau chaude & 143 & 69,08 \\
\hline & Autres & 64 & 30,92 \\
\hline \multicolumn{4}{|c|}{ Caractéristiques cliniques } \\
\hline \multirow{2}{*}{$\begin{array}{l}\text { Délai d'admission (Heures) } \\
(n=211)\end{array}$} & {$[0-6[$} & 149 & 70,62 \\
\hline & {$[6-24[$} & 62 & 29,38 \\
\hline \multirow{2}{*}{$\begin{array}{l}\text { Surface corporelle brûlée } \\
(\%) \\
(n=211)\end{array}$} & [0 à 20] & 103 & 48,82 \\
\hline & ] 20 à 100] & 108 & 51,18 \\
\hline \multirow{2}{*}{$\begin{array}{l}\text { Profondeur } \\
\text { de la brûlure } \\
(n=212)\end{array}$} & $2^{\mathrm{e}}$ degré superficiel & 195 & 91,98 \\
\hline & $2^{\mathrm{e}}$ degré profond $/ 3^{\mathrm{e}}$ degré & 17 & 8,02 \\
\hline \multirow{2}{*}{$\begin{array}{l}\text { Evolution } \\
(n=212)\end{array}$} & Guérison & 176 & 83,02 \\
\hline & Décès & 36 & 16,98 \\
\hline
\end{tabular}


Tableau 3 : Profil des perturbations de l'ionogramme sanguin.

\begin{tabular}{|c|c|c|c|c|c|}
\hline $\begin{array}{l}\text { Paramètres } \\
\text { de l'ionogramme } \\
\text { sanguin }\end{array}$ & Perturbations & & $\begin{array}{r}\text { A l'entrée } \\
\text { Effectif }(\%)\end{array}$ & & $\begin{array}{l}\text { Au contrôle } \\
\text { Effectif }(\%)\end{array}$ \\
\hline Sodium & $\begin{array}{l}\text { Hypernatrémie } \\
\text { Hyponatrémie }\end{array}$ & $(n=208)$ & $\begin{array}{r}8(3,85) \\
58(\mathbf{2 7 , 8 8})\end{array}$ & $(n=67)$ & $\begin{array}{r}2(2,90) \\
17(\mathbf{2 4 , 6 4 )}\end{array}$ \\
\hline Potassium & $\begin{array}{l}\text { Hyperkaliémie } \\
\text { Hypokaliémie }\end{array}$ & $(n=209)$ & $\begin{array}{r}13(6,22) \\
6(2,87)\end{array}$ & $(n=69)$ & $\begin{array}{l}7(10,14) \\
7(10,14)\end{array}$ \\
\hline Calcium & $\begin{array}{l}\text { Hypercalcémie } \\
\text { Hypocalcémie }\end{array}$ & $(n=210)$ & $\begin{array}{l}46(21,90) \\
36(17,14)\end{array}$ & $(n=69)$ & $\begin{array}{l}15(21,74) \\
21(\mathbf{3 0 , 4 3 )}\end{array}$ \\
\hline Magnésium & $\begin{array}{l}\text { Hypermagnésémie } \\
\text { Hypomagnésémie }\end{array}$ & $(n=200)$ & $\begin{array}{l}48(24,00) \\
47(23,50)\end{array}$ & $(n=66)$ & $\begin{array}{r}8(12,12) \\
18(\mathbf{2 7}, 27)\end{array}$ \\
\hline Bicarbonates & $\begin{array}{l}\text { Hyperbicarbonatémie } \\
\text { Hypobicarbonatémie }\end{array}$ & $(n=152)$ & $\begin{array}{r}2(1,32) \\
82(\mathbf{5 3}, \mathbf{9 5})\end{array}$ & $(n=50)$ & $\begin{array}{r}2(4,00) \\
16(\mathbf{3 2 , 0 0 )}\end{array}$ \\
\hline Phosphates & $\begin{array}{l}\text { Hyperphosphatémie } \\
\text { Hypophosphatémie }\end{array}$ & $(n=189)$ & $\begin{array}{l}54(28,57) \\
35(18,52)\end{array}$ & $(n=66)$ & $\begin{array}{l}19(\mathbf{2 8 , 7 9 )} \\
12(18,18)\end{array}$ \\
\hline Chlore & $\begin{array}{l}\text { Hyperchlorémie } \\
\text { Hypochlorémie }\end{array}$ & $(n=209)$ & $\begin{array}{r}112(\mathbf{5 3}, \mathbf{5 9}) \\
6(2,87)\end{array}$ & $(n=68)$ & $\begin{array}{r}27(\mathbf{3 9}, 71) \\
3(4,41)\end{array}$ \\
\hline Protéines totales & $\begin{array}{l}\text { Hyperprotidémie } \\
\text { Hypoprotidémie }\end{array}$ & $(n=210)$ & $\begin{array}{l}31(14,76) \\
56(\mathbf{2 6 , 6 7}) \\
\end{array}$ & $(n=69)$ & $\begin{array}{r}2(2,90) \\
43(\mathbf{6 2}, \mathbf{3 2}) \\
\end{array}$ \\
\hline
\end{tabular}

Tableau 4 : Profil des paramètres d'entrée de l'ionogramme sanguin en fonction du délai d'admission et de l'évolution clinique du patient.

\begin{tabular}{lcccccc}
\hline $\begin{array}{l}\text { Paramètres } \\
\text { de l'ionogramme }\end{array}$ sanguin & \multicolumn{2}{c}{ Délai d'admission } & $\begin{array}{c}\text { p- } \\
\text { value }\end{array}$ & \multicolumn{2}{c}{ Evolution du patient } & p-value \\
& $\begin{array}{c}\mathbf{0 - 6} \text { heures } \\
(\mathbf{n = 1 4 8})\end{array}$ & $\begin{array}{c}\mathbf{6 - 2 4} \text { heures } \\
(\mathbf{n = 5 9 )}\end{array}$ & & $\begin{array}{c}\text { Vivants } \\
(\mathbf{n = 1 7 6})\end{array}$ & $\begin{array}{c}\text { Décédés } \\
(\mathbf{n = 3 6})\end{array}$ & \\
\hline Sodium (mmol/L) & $135,80 \pm 6,64$ & $135,33 \pm 5,49$ & 0,6315 & $136,45 \pm 5,54$ & $131,83 \pm 8,31$ & $<\mathbf{0 , 0 0 0}$ \\
Potassium (mmol/L) & $4,85 \pm 0,92$ & $4,90 \pm 0,85$ & 0,7535 & $4,81 \pm 0,86$ & $5,12 \pm 1,02$ & 0,0570 \\
Calcium (mmol/L) & $2,49 \pm 0,45$ & $2,42 \pm 0,44$ & 0,2870 & $2,49 \pm 0,44$ & $2,38 \pm 0,48$ & 0,1802 \\
Magnésium (mmol/L) & $0,82 \pm 0,70$ & $0,74 \pm 0,26$ & 0,3633 & $0,82 \pm 0,66$ & $0,72 \pm 0,25$ & 0,4005 \\
Bicarbonates (mmol/L) & $16,26 \pm 4,04$ & $16,53 \pm 3,41$ & 0,6958 & $16,74 \pm 3,74$ & $14,81 \pm 3,89$ & $\mathbf{0 , 0 0 6 0}$ \\
Phosphates (mmol/L) & $1,74 \pm 0,53$ & $1,75 \pm 0,55$ & 0,8967 & $1,76 \pm 0,50$ & $1,65 \pm 0,69$ & 0,2786 \\
Chlore (mmol/L) & $108,85 \pm 6,40$ & $106,89 \pm 5,40$ & $\mathbf{0 , 0 1 8 3}$ & $108,28 \pm 6,11$ & $108,19 \pm 6,51$ & 0,9369 \\
Protéines totales (g/L) & $63,23 \pm 13,36$ & $58,43 \pm 12,32$ & $\mathbf{0 , 0 0 8 7}$ & $63,06 \pm 12,58$ & $55,68 \pm 14,60$ & $<\mathbf{0 , 0 0 1 1}$ \\
\hline
\end{tabular}


Tableau 5 : Profil des paramètres d'entrée de l'ionogramme sanguin en fonction de la surface corporelle brûlée et de la profondeur de la brûlure.

\begin{tabular}{|c|c|c|c|c|c|c|}
\hline \multirow{2}{*}{$\begin{array}{l}\text { Paramètres } \\
\text { de l'ionogramme } \\
\text { sanguin }\end{array}$} & \multicolumn{3}{|c|}{ Surface corporelle brûlée } & \multicolumn{3}{|c|}{ Profondeur de la brûlure } \\
\hline & $0-20 \%$ & $20-100 \%$ & p-value & $\begin{array}{l}2^{\text {ème }} \text { degré } \\
\text { superficiel }\end{array}$ & $\begin{array}{r}2^{\text {ème }} \text { degré } \\
\text { profond / } 3^{\text {ème }} \\
\text { degré }\end{array}$ & p-value \\
\hline Sodium (mmol/L) & $136,74 \pm 5,30$ & $134,67 \pm 7,02$ & $\mathbf{0 , 0 0 8 9}$ & $136,96 \pm 6,02$ & $135,56 \pm 6,34$ & 0,3961 \\
\hline Potassium (mmol/L) & $4,71 \pm 0,77$ & $5,01 \pm 0,98$ & 0,0067 & $4,74 \pm 1,23$ & $4,87 \pm 0,86$ & 0,5549 \\
\hline Calcium (mmol/L) & $2,57 \pm 0,44$ & $2,37 \pm 0,43$ & 0,0008 & $2,46 \pm 0,36$ & $2,47 \pm 0,45$ & 0,9373 \\
\hline Magnésium (mmol/L) & $0,75 \pm 0,24$ & $0,84 \pm 0,82$ & 0,2757 & $0,66 \pm 0,31$ & $0,81 \pm 0,63$ & 0,3717 \\
\hline Bicarbonates $(\mathrm{mmol} / \mathrm{L})$ & $16,95 \pm 3,88$ & $15,70 \pm 3,71$ & $\mathbf{0 , 0 2 2 5}$ & $14,88 \pm 4,16$ & $16,42 \pm 3,82$ & 0,2680 \\
\hline Phosphates (mmol/L) & $1,73 \pm 0,51$ & $1,74 \pm 0,56$ & 0,9116 & $1,62 \pm 0,59$ & $1,75 \pm 0,53$ & 0,3843 \\
\hline Chlore $(\mathrm{mmol} / \mathrm{L})$ & $108,16 \pm 6,70$ & $108,36 \pm 5,64$ & 0,8146 & $106,48 \pm 6,04$ & $108,41 \pm 6,17$ & 0,2280 \\
\hline Protéines totales $(\mathrm{g} / \mathrm{L}$ ) & $63,88 \pm 12,23$ & $59,79 \pm 13,85$ & $\mathbf{0 , 0 1 2 3}$ & $61,38 \pm 16,05$ & $61,83 \pm 13,00$ & 0,8942 \\
\hline
\end{tabular}

\section{DISCUSSION}

Les brûlures infantiles représentent un fardeau pour les services de santé, particulièrement dans les pays à ressources limitées. Notre étude visait à déterminer les troubles hydro-électrolytiques occasionnés par des brûlures chez les enfants pris en charge au CHUP-CDG. Parmi les 7908 patients hospitalisés dans le service de chirurgie du CHUP-CDG, le nombre d'enfants brûlés a été de 384 sur une période de 2,5 ans, soit une fréquence de 4,85\% des hospitalisés par an, avec un pic essentiellement durant la période froide (janvier-février).

Ces données viennent confirmer celles de Guibla (2015) et Konvolbo (2011) au Burkina Faso, qui retrouvaient des fréquences respectives de 5,1 et $4,78 \%$; de même que Adelin (Adelin et al., 2019) au Bénin (4,52\%). Ces auteurs soulignaient de plus dans leurs études, la survenue des brûlures surtout en période froide. Othman (Othman et al., 2015) en Irak et Zahid au Maroc (Zahid et al., 2011) rapportaient également que les enfants se brûlaient plus en hiver. Cette augmentation durant cette période pourrait s'expliquer dans notre contexte par la nécessité de chauffer plus souvent l'eau pour la toilette et à l'utilisation du feu de bois pour le chauffage en vue de lutter contre le froid, favorisant ainsi la survenue de brûlures. D'ailleurs, tous nos patients étaient victimes de brûlure thermique; plus particulièrement avec des liquides chauds $(84,6 \%)$. Ce constat pose l'épineux problème de la responsabilité des parents en ce qui concerne la surveillance des enfants et la nécessité d'adopter des mesures de sécurité afin de limiter au maximum la survenue de tels accidents.

Ces brûlures survenaient surtout chez les enfants de moins de 5 ans, avec un âge moyen de 38,84 mois (3,23 ans). Parmi ces enfants, les garçons représentaient $53,47 \%$ des cas, avec un sex ratio à 1,14 . Nos résultats sont 
semblables à ceux de Adelin, qui notait 71,4\% d'enfants de moins de 5 ans, un âge moyen de 3,5 ans et $57,1 \%$ de garçons (sex-ratio de 1,3) dans son étude au Bénin (Adelin et al., 2019). Cela s'expliquerait par le fait que la petite enfance constitue une période où l'enfant découvre son environnement avec une insouciance et une absence d'évaluation du danger, l'exposant à de multiples risques. Aussi, la prédominance masculine pourrait s'expliquer par le comportement des garçons, décrit comme étant plus hyperactif.

Dans notre étude, les patients ont été admis dans un délai de 24 heures avec 74,26\% de consultation dans les six premières heures. Guedehounsou (2015) au Burkina Faso avait également trouvé que $61,74 \%$ des brûlés étaient admis dans les six premières heures. Par contre, Zahid (Zahid et al., 2011) au Maroc et Bagayoko (2007) au Mali, notaient respectivement $34,5 \%$ et $20 \%$ de cas reçus dans un délai de moins de six heures. Ce constat pourrait être dû au niveau d'instruction des parents plus élevé en milieu urbain, comme cela était le cas des patients de notre étude ; ne tardant donc pas à consulter pour des soins spécialisés.

Dans le cadre du bilan paraclinique réalisé chez les enfants à l'admission, l'ionogramme sanguin a permis de mettre en évidence des troubles hydroélectrolytiques divers. Ainsi, nous avons noté une hyponatrémie, une hypobicarbonatémie, une hypoprotidémie et une hyperchlorémie (Tableau 3). Une hyponatrémie était retrouvée à l'admission par Konvolbo (2011) au Burkina Faso $(18,4 \%)$ et par Hauhouot-attoungbre (Hauhouot-Attoungbre et al., 2005) en Côte d'ivoire (14\%). De même, Hauhouotattoungbre (Hauhouot-Attoungbre et al., 2005) retrouvait à $\mathrm{J} 0$ une hypoprotidémie dans $73 \%$ de cas. L'hyponatrémie est un phénomène courant chez les personnes brûlés et pourrait s'expliquer par l'épuisement du sodium extracellulaire à la suite des modifications de la perméabilité capillaire (Ramos, 2000). L'hypoprotidémie pourrait être due à la dénaturation des protéines par la chaleur et à l'hyperperméabilité capillaire secondaire à la brûlure, entrainant une fuite protéique (Ramos,
2000; Hauhouot-Attoungbre et al., 2005). En effet, la physiopathologie de la brûlure fait état d'une phase aigüe ou phase de déséquilibre hémodynamique, dominée par des troubles hydroélectrolytiques majeurs et une phase d'hypercatabolisme. Quelle que soit l'étiologie, la brûlure provoque une destruction d'un certain nombre de cellules, de capillaires superficiels et d'ailleurs de nombreuses études se sont intéressées au processus de cicatrisation (Metowogo et al., 2020; Sene et al., 2020). Les zones brûlées sont sujettes à un œdème massif avec ischémie et augmentation de la perméabilité capillaire et cellulaire avec fuite massive d'eau, de protéines et d'électrolytes. Cette réaction inflammatoire d'abord locale, va se généraliser par la suite en touchant les tissus sains quand la surface de la brûlure dépasse $20 \%$. Ces perturbations sont brutales, d'emblée maximales entraînant une fuite liquidienne intense, mais de durée relativement brève. Ainsi, hyponatrémie et hypoprotidémie pourraient donc être expliquées par ces fuites ioniques et protéiques, dont l'intensité est fonction de l'étendue de la brûlure. Ces pertes exsudatives pourraient également justifier une perte de bicarbonates. De plus, l'hypobicarbonatémie observée dans notre étude, de même que l'hyperchlorémie, pourraient s'expliquer par l'insuffisance rénale aiguë que peut engendrer la brûlure. En effet, Les bouleversements hydriques initiaux, ainsi que les défaillances multiviscérales secondaires, peuvent altérer la fonction rénale. L'apparition d'une insuffisance rénale aiguë (IRA) a toujours été péjorative et associée à un mauvais pronostic vital chez les brûlés graves. Plusieurs mécanismes physiopathologiques pourraient expliquer l'IRA lors des brûlures, mais le principal est représenté par l'hypovolémie à la phase aigüe, pouvant se compliquer de nécrose tubulaire aigüe (Bargues et al., 2009).

$\mathrm{Au}$ contrôle, certains troubles hydroélectrolytiques étaient toujours présents : l'hyponatrémie, l'hypobicarbonatémie, l'hypoprotidémie et l'hyperchlorémie. S'y sont associées une hypocalcémie, une hypomagnésémie et une hyperphosphatémie (Tableau 3). Hauhouot-attoungbre notifiait 
dans son étude en Côte d'ivoire, une hypoprotidémie $(100 \%)$, une hypocalcémie $(100 \%)$, une hyponatrémie $(50 \%)$ et une hypomagnésémie (28\%). Classiquement, l'hypoprotidémie et l'hypocalcémie concomitante sont des perturbations communes dans la brûlure et sont souvent majorées au cours de l'évolution, chez l'enfant comme chez l'adulte (Hauhouot-Attoungbre et al., 2005). L'hypoprotidémie, comme précédemment décrit, pourrait être due à la dénaturation protéique par la chaleur mais aussi par le passage des protéines dans le milieu extravasculaire suite à l'hyperperméabilité capillaire consécutive à la brûlure. De plus, elle pourrait être majorée par la dilution consécutive au remplissage vasculaire. L'hypocalcémie serait due à l'hypoprotidémie, puisqu'une fraction importante du calcium est fixée aux protéines, notamment à la sérum albumine. En outre, chez le brûlé de plus de $40 \%$ de SCB une albuminémie inférieure à $20 \mathrm{~g} / \mathrm{L}$ est de règle. En ce qui concerne les paramètres tels que la phosphatémie, la chlorémie et la magnésémie, la physiopathologie de la brûlure ne fait pas expressément cas de ces ions. Cependant l'hyperphosphatémie pourrait s'expliquer par l'hypercatabolisme consécutif à la brûlure. En effet, l'accélération du catabolisme tissulaire peut entrainer une hyperphosphatémie (Marshall et al., 2005).

L'étude du profil des paramètres d'entrée de l'ionogramme sanguin en fonction des caractéristiques cliniques des patients nous a permis de mettre en évidence qu'une hypochlorémie et une hypoprotidémie étaient significativement associées à un délai d'admission supérieur à six heures. La physiopathologie de l'hypoprotidémie au cours de la brûlure est bien établie ; elle apparait dans les premières heures suivant la brûlure et peut s'aggraver au cours de l'évolution; justifiant que les patients de notre étude reçus au-delà de six heures aient une protidémie plus basse (Hauhouot-Attoungbre et al., 2005). De même, une hyponatrémie, une hyperkaliémie, une hypocalcémie, une hypobicarbonatémie et une hypoprotidémie étaient significativement associées à une importante surface corporelle brûlée. Ces troubles ioniques objectivés dans notre étude pourraient s'expliquer par les conséquences physiopathologiques de la brûlure; lesquelles sont liées à la gravité de la brûlure estimée par l'étendue de la SCB. Aucun paramètre de l'ionogramme sanguin n'a présenté une variation significative en fonction de la profondeur de la brûlure. Enfin, la natrémie, la bicarbonatémie et la protidémie des enfants décédés étaient significativement plus basses à l'entrée, comparativement aux survivants. La sévérité de l'hyponatrémie a été établie par certains auteurs comme étant un facteur pronostique indépendant prédictif de mortalité. De plus, elle pourrait accroitre la mortalité chez les patients en étant à l'origine de dysfonctions d'organes (Bennani et al., 2003).

\section{Conclusion}

Les brûlures de l'enfant restent une pathologie fréquente et préoccupante. Au cours de notre étude, nous avons pu mettre en évidence diverses perturbations des paramètres de l'ionogramme sanguin. Lors de l'admission des patients, il s'agissait essentiellement d'une hyponatrémie, une hypobicarbonatémie, une hyperchlorémie et une hypoprotidémie. La prise en charge précoce et adaptée de ces troubles est nécessaire pour améliorer le pronostic. La mortalité dans notre étude était de $16,98 \%$; soulignant encore l'intérêt de la prévention primaire via l'information, l'éducation et la sensibilisation.

\section{CONFLIT D'INTERETS}

Les auteurs déclarent ne pas avoir de conflit d'intérêts.

\section{CONTRIBUTIONS DES AUTEURS}

FMS, ST et JS ont initié l'étude et rédigé le protocole. ST a collecté les données. AK, DS et ST ont effectué les dosages. FMS a analysé les données et rédigé le manuscrit. AK, AK et RK ont corrigé le manuscrit. EK et JS ont validé la version finale.

\section{REMERCIEMENTS}

Les auteurs remercient le personnel du service de chirurgie et les technologistes 
biomédicaux du service des laboratoires du CHU pédiatrique Charles de Gaulle de Ouagadougou.

\section{REFERENCES}

Adelin TB, Frédéric TNC, Patrice HCY, Affiémin AC. 2019. Prise en Charge des Brûlures Graves de l'enfant en Réanimation à l'hôpital Universitaire de Parakou au Bénin. European Scientific Journal, 15(3): 199-209. DOI: https://doi.org/10.19044/esj.2019.v15n3p 199

Bagayoko A. 2007. Etude des Brûlures graves dans le service de la chirurgie pédiatrique. Thèse de Doctorat, Université de Bamako, Bamako, $120 \mathrm{p}$.

Bargues L, Leclerc T, Donat N, Jault P. 2009. Conséquences systémiques des brûlures étendues. Réanimation, 18(8): 687-693. DOI:

https://doi.org/10.1016/j.reaurg.2009.09. 005

Bennani SL, Abouqal R, Zeggwagh AA, Madani N, Abidi K, Zekraoui A, Kerkeb O. 2003. Incidence, étiologies et facteurs pronostiques de l'hyponatrémie en réanimation. La Revue de Médecine Interne, 24(4): 224-229. DOI: https://doi.org/10.1016/S02488663(02)00811-1

Beyiha G, Binam F, Batamack JF, Sosso MA. 2000. Traitement et pronostic de la brûlure grave au centre des grands brûlés de Douala, Cameroun. Annals of Burns and Fire Disasters, 13(3): 131-137.

Fadeyibi IO, Mustapha IA, Ibrahim NA, Faduyile FI, Faboya MO, Jewo PI, Ademiluyi SA. 2011. Characteristics of paediatric burns seen at a tertiary centre in a low income country: A five year (2004-2008) study. Burns, 37(3): 528534.

DOI: https://doi.org/10.1016/j.burns.2010.09.0 15

Guedehounsou L. 2019. Les brûlures chez l'enfant: Epidémiologie et facteurs pronostiques au CHUP-CDG : à propos de 472 cas. Thèse de Doctorat, Université Joseph Ki-Zerbo, Ouagadougou, 161 p.
Guibla I. 2015. Prise en charge des brûlures graves dans le service de réanimation polyvalente du CHU Souro Sanou de Bobo-Dioulasso. Thèse de Doctorat, Université Joseph Ki-Zerbo, Ouagadougou, $175 \mathrm{p}$.

Gülhan B, Kanık YS, Hayran M, Özkaya PA, Güney D, Akın KG, Kaygısız H, Tezer H, Şenel E. 2019. Infections in Pediatric Burn Patients: An Analysis of One Hundred Eighty-One Patients. Surgical Infections, 21(4): 357-362. DOI: https://doi.org/10.1089/sur.2019.010

Hauhouot-Attoungbre ML, Mlan WCH, Edjeme NA, Ahibo H, Vilasco B, Monnet D. 2005. Intérêt du ionogramme chez le brûlé thermique grave. Annales de Biologie Clinique, 63(4): 417-421.

Konvolbo JW. 2011. Brûlure de l'enfant au Centre Hospitalier Universitaire Pédiatrique Charles de Gaulle au Burkina Faso. Thèse de Doctorat, Université Joseph Ki-Zerbo, Ouagadougou, 106 p.

Marshall WJ, Bangert SK, Raynaud E. 2005. Biochimie Médicale: Physiopathologie et Diagnostic. Elsevier / Masson : Paris.

Metowogo K, Fankibe N, Kantati YT, Adi K, Darré T, Eklu-Gadegbeku K, Aklikokou KA. 2020. Burn wound healing effects of the root hydroethanolic extract of Cochlospermum planchonii in mice. International Journal of Biological and Chemical Sciences, 14(9): 3275-3284. DOI:

https://doi.org/10.4314/ijbcs.v14i9.24

Minkandé Z, Beyiha G, Ella A, Sosso M, Binam F, Chiabi A. 2004. Brûlures graves chez l'enfant: Aspects épidémiologiques et thérapeutiques à l'Hôpital GynécoObstétrique et Pédiatrique de Yaoundé. Clinics in Mother and Child Health, 1(2): 85-88.

OMS. 2020. Organisation Mondiale de la Santé. Brûlures. OMS, Suisse. Disponible sur https://www.who.int/fr/newsroom/fact-sheets/detail/burns

Othman N, Kendrick D, Al-Windi A. 2015. Childhood burns in Sulaimaniyah province, Iraqi Kurdistan: A prospective study of admissions and outpatients. 
Burns, 41(2): 394-400. DOI: https://doi.org/10.1016/j.burns.2014.07.0 08

Peck MD. 2011. Epidemiology of burns throughout the world. Part I: Distribution and risk factors. Burns, 37(7): 1087-1100. DOI:

https://doi.org/10.1016/j.burns.2011.06.0 05

Ramos CG. 2000. Management of fluid and electrolyte disturbances in the burn patient. Annals of Burns and Fire Disasters, 13(4): 201-205.

Ryder C, Mackean T, Hunter K, Towers K, Rogers K, Holland AJA, Ivers R. 2020. Factors contributing to longer length of stay in Aboriginal and Torres Strait Islander children hospitalised for burn injury. Injury Epidemiology, 7(1): 52. DOI: https://doi.org/10.1186/s40621020-00278-7

Santiso L, Tapking C, Lee JO, Zapata-Sirvent R, Pittelli CA, Suman OE. 2020. The Epidemiology of Burns in Children in Guatemala: A Single Center Report. Journal of Burn Care \& Research, 41(2): 248-253.

DOI: https://doi.org/10.1093/jbcr/irz157

Sene M, Barboza FS, Top B, Ndiaye M, Sarr A, Fall AD, Sy GY. 2020. Activité cicatrisante de l'extrait aqueux des feuilles de Elaeis guineensis Jacq. (Arecaceae). International Journal of
Biological and Chemical Sciences, 14(3): 674-684.

DOI: https://doi.org/10.4314/ijbcs.v14i3.3

SFETB. 2008. Société française d'étude et de traitement des brûlures. Rapport annuel concernant l'épidémiologie de la brûlure en France métropolitaine, SFETB. Disponible sur http://www.sfetb.org/index.php?rub=epi demiologie.

Wassermann D. 2002. Critères de gravité des brûlures. Épidémiologie, prévention, organisation de la prise en charge. Pathologie Biologie, 50(2): 65-73. DOI: https://doi.org/10.1016/S03698114(01)00271-1

Zahid A, Atannaz J, Alaoui M, Rafik A, EzzoubiM, Diouri M, Chlihi A, BahecharN, Boukind EH. 2011. Profil épidémiologique des enfants brûlures admis au centre national des brûlés, Maroc. Ann. Burns Fire Disasters, 24:171-174.

Zhang C, Chang M, Zhou Z, Yi L, Huang X, Gao C, Guo F, Huan J. 2020. Factors Influencing Length of Hospital Stay and Predictors Affecting Probability of Requiring Surgery in Severely Pediatric Burn Patients. Journal of Burn Care \& Research: Official Publication of the American Burn Association, 41(6): 11651171.

DOI: https://doi.org/10.1093/jbcr/irz202 